\title{
An observational cohort study on impact, dimensions and outcome of perceived fatigue in adult 5q-spinal muscular atrophy patients receiving nusinersen treatment
}

\author{
Camilla Binz ${ }^{1}$. Olivia Schreiber-Katz ${ }^{1} \cdot$ Mareike Kumpe $^{1} \cdot$ Gresa Ranxha $^{1} \cdot$ Hannah Siegler ${ }^{1} \cdot$ Gary Wieselmann $^{1}$. \\ Susanne Petri ${ }^{1} \cdot$ Alma Osmanovic $^{1}$
}

Received: 26 June 2020 / Revised: 10 September 2020 / Accepted: 12 September 2020 / Published online: 7 October 2020

(c) The Author(s) 2020

\begin{abstract}
Background Efficacy of nusinersen in adult 5q-spinal muscular atrophy (SMA) patients regarding motor function has recently been demonstrated. However, additional outcome measures are needed to capture non-motor improvements. Fatigue is a common and disabling symptom in neurologic diseases, but little is known about its frequency, characteristics and associated factors in SMA.

Objective To characterize fatigue in SMA patients receiving nusinersen, identify associated factors and evaluate fatigue as potential patient-reported outcome measure (PRO).

Methods We assessed fatigue in adults with genetically confirmed 5q-SMA in a prospective longitudinal monocentric study using the Fatigue Severity Scale (FSS) and the Multidimensional Fatigue Inventory (MFI). Factors associated with fatigue including health-related quality of life (HRQOL) were evaluated.

Results $75 \%$ of participants were abnormally fatigued with highest scores in the dimensions physical, followed by general fatigue and reduced activity. 53\% agreed that fatigue was among their three most disabling symptoms. Reduced activity was reported more extensively by participants with $\geq 4$ copies of the survival of motor neuron 2 gene and better motor function. General and mental fatigue correlated positively with age and disease duration. HRQOL was inversely correlated with physical fatigue, which was not associated with disease or participant characteristics. During 14 months of nusinersen treatment, fatigue measures remained mostly stable with a trend towards improvement in reduced activity, general and physical fatigue. Conclusion Fatigue is a frequent and relevant complaint in adult SMA patients. Fatigue should be taken into consideration as additional outcome measure, but needs further evaluation in a larger patient cohort over a longer observation period.
\end{abstract}

Keywords Fatigue $\cdot$ Fatigue severity scale (FSS) $\cdot$ Multidimensional fatigue inventory (MFI) $\cdot$ Spinal muscular atrophy $(\mathrm{SMA}) \cdot$ Nusinersen $\cdot$ Patient-reported outcome measure (PRO)

Camilla Binz and Olivia Schreiber-Katz contributed equally as first authors to the manuscript

Susanne Petri and Alma Osmanovic contributed equally as senior authors to the manuscript

Olivia Schreiber-Katz

schreiber-katz.olivia@mh-hannover.de

Extended author information available on the last page of the article

\section{Introduction}

Spinal muscular atrophy (SMA) is an inherited neuromuscular disorder, mainly caused by homozygous mutations in the survival of motor neuron (SMN) 1 gene on chromosome $5 \mathrm{q}$ [1]. The resulting lack of SMN protein leads to a degeneration of alpha motor neurons in spinal cord and brain stem. Patients suffer from progressive muscle weakness, scoliosis, contractures and respiratory insufficiency. Different SMA subtypes can be distinguished based on the age at symptom onset and the best motor milestones achieved. These phenotypes span a broad continuum, ranging from SMA type 0 with prenatal manifestation of hypotonia and respiratory failure at birth, to SMA type IV with development of muscle 
weakness in the second or third decade of life and normal life expectancy [2]. The dosage of $S M N 2$, a neighboring nearly identical gene, has been identified as a potent modifier of SMA severity: a copy number of $S M N 2$ of $\geq 4$ is highly predictive of achieving the ability to walk (SMA type III/ IV) [3].

Since the broad approval of nusinersen, an antisense oligonucleotide which increases SMN protein production by modification of $S M N 2$ pre-mRNA splicing, a causative treatment option for SMA does exist. Pivotal trials were conducted in infants and children [4, 5]. Only recently, safety and feasibility of administration in older SMA patients has been demonstrated [6, 7]. First studies indicated beneficial treatment effects in adults and more data on long-term efficacy are soon to be expected [8-11]. It is controversial if classical motor function outcome measures are sensitive enough in patients with severely or very mildly impaired motor function, as floor and ceiling effects may occur [12, 13]. Further, motor function changes in severely affected patients may be small, but can have importance for the individual patient. Thus, facing the development of novel therapeutic options, alternative outcome measures besides motor function are needed.

Fatigue is a commonly reported symptom in neurological and especially neuromuscular disorders with an estimated prevalence of severe fatigue of $60 \%$ [14]. There is no exact definition of fatigue, but it is often described as "overwhelming sense of tiredness, lack of energy and feeling of exhaustion, mental, physical or both" [15]. Further, patients report an increasing sense of effort and a perceived mismatch between effort and actual performance or exhaustion. In contrast, the concept of fatigability (sometimes also referred to as "peripheral" fatigue) describes the decline in performance over time. Establishing a relationship between fatigue and fatigability has proven difficult [16].

Fatigue is believed to be a primary symptom of neurological disorders and not secondary to medication, laboratory abnormalities, mood or sleep disturbances. The prevalence of fatigue exceeds the - on the basis of age and disability-expected levels and, generally, fatigue does not correlate with the nature or severity of the underlying disease [14]. Regarding neuromuscular diseases, fatigue has so far been characterized in myasthenia gravis [17], postpolio syndrome [18, 19], congenital myopathies [20] and amyotrophic lateral sclerosis [21]. In SMA, fatigue was described early [22] and, from our clinical experience, is a frequent and burdening symptom. Surprisingly, there is little and sometimes even contradicting information on prevalence of and factors associated with fatigue in SMA [23-25]. Studies focusing on the several dimensions of fatigue and its impact on SMA patients, particularly in adults, are lacking.
In this study, we aimed to characterize perceived fatigue in adult SMA and identify associated factors. We further hypothesized that fatigue impacts quality of life and might be a suitable additional patient-reported outcome measure (PRO) in clinical trials or post-marketing surveillance.

\section{Methods}

\section{Participants}

SMA patients aged 18 years and above who received their first nusinersen administration were enrolled in a prospective longitudinal monocentric observational cohort study at the Department of Neurology of Hannover Medical School between April 2018 and October 2019. All had a genetically confirmed diagnosis of 5q-SMA (homozygous deletion of exon 7 or/and exon 8 of $S M N 1$ ). All participants received nusinersen according to the approved scheme on days $0,14,28,63$ and every four months after completing the medication loading period. Our total SMA cohort consisted of 28 patients, 19 of whom received their first nusinersen administration at our facility and were, therefore, identified as potentially eligible. Complete baseline characteristics were available for 18 patients who were confirmed eligible and enrolled. 15 participants completed all questionnaires and assessments at the first nusinersen administration, one participant only completed MFI and not FSS. Reasons for refusal to fill in questionnaires were the impression of redundancy within questionnaires $(N=1)$ or not specified $(N=2)$. Follow-up data were collected during inpatient stays for nusinersen treatment. There was no loss to follow-up which minimized the risk of attrition bias. 14 months of follow-up data since therapy initiation were available for 14 participants, as two participants had not yet completed 14 months' nusinersen administration at the time of data analysis and two had not completed the questionnaires after month 10. Reasons for non-completion of questionnaires at month 10 were specified as impression of redundancy $(N=1)$ and lack of time $(N=1)$. All collected data from all participants were included into the statistical analyzes.

Perceived fatigue and health-related quality of life were assessed using standardized questionnaires prior to and during treatment. Socio-demographic and disease characteristics including motor function were obtained from medical records or assessed at first administration.

\section{Fatigue measures}

Participants completed the German Version of the Fatigue Severity Scale (FSS) and Multidimensional Fatigue Inventory (MFI) at day 0 (baseline), day 63 (month 2), month 6 , month 10 and month 14 . The FSS is one of 
the most frequently used unidimensional fatigue scales, which was initially developed for multiple sclerosis and systemic lupus erythemathodes $[15,26]$. It is applicable in neuromuscular diseases and has been validated for SMA patients [20, 27]. It is a self-reported questionnaire and measures the impact of fatigue in the time frame of the "past week". The scale consists of nine items with a seven point Likert-type scale. A mean score is calculated (ranging from one to seven), whereas a mean score of more than four is considered as abnormal fatigue and a score above five indicates severe fatigue [28, 29]. In case of a missing answer to an FSS item, the missing value was substituted with the mean of the remaining items [30].

As no other fatigue scales have been validated in SMA patients so far, we chose the MFI to measure the different dimensions of perceived fatigue. It is also a self-report instrument and contains 20 items which are categorized in five dimensions: general fatigue, physical fatigue, mental fatigue, reduced motivation and reduced activity [31]. The MFI measures how a patient felt "lately" on a fivepoint Likert-type scale. For each dimension, a total score is calculated, which can range from four to 20 while higher scores indicate increased fatigue. As fatigue measured by MFI is dependent on age and sex, cut-off values that indicate abnormal fatigue were defined as the respective 75th percentile of the German population according to Singer et al. (males: aged $\leq 39$ years cut-off $\geq 8,40-59$ years cutoff $\geq 10, \geq 60$ years cut-off $\geq 13$; females: aged $\leq 39$ years cut-off $\geq 10,40-59$ years cut-off $\geq 11, \geq 60$ years cutoff $\geq 13$ ) [32]. Missing answers were replaced with the mean of the respondent's completed answers within the same subscale as previously described [33].

\section{Health-related quality of life measures (HRQOL)}

Health state was measured with the German version of the EuroQol Five Dimension Five Level Scale (EQ-5D-5L), a standardized measure of HRQOL in adults [34, 35]. It captures five dimensions: mobility, self-care, usual activities, pain/discomfort and anxiety/depression. Each dimension is scaled in five levels of perceived problems $($ level one $=$ no problems, level five $=$ extreme problems) and together they result in a distinct health state (11111-55555). Those health states were converted into index values using the value set derived from the German reference sample according to the provider's instructions $[30,36]$. Additionally, the EQ-5D-5L comprises a vertical visual analog scale (VAS) scored from 0 to 100 , capturing the "health state today" (higher scores indicate better HRQOL). The EQ-5D-5L has been validated in Europe and is frequently used in rare diseases, such as SMA [37, 38].

\section{Motor function measures}

Motor function was assessed by professional physiotherapists using the Six-Minute Walk Test (6MWT), Hammersmith Functional Motor Scale Expanded (HFMSE) and Revised Upper Limb Module (RULM). The 6MWT assesses the submaximal aerobic capacity and has been found reliable and valid in ambulatory SMA patients [39]. The HFMSE is a disease-specific scale developed for SMA type II and III patients with ambulatory difficulties. It measures gross motor function using 33 items and calculating a sum score of up to 66 points (higher scores represent a better function) [40, 41]. The RULM, another disease-specific scale, measures function of upper extremities and performance in activities of daily living. It consists of 20 items with a maximum sum score of 37 points (higher scores indicate a better function) [42].

\section{Statistics}

Statistical analysis was performed using IBM ${ }^{\circledR}$ Statistical Software Package of Social Science (SPSS ${ }^{\circledR}$, Chicago, IL, USA) version 26. For all analyzes, significance levels were set at $p<0.05$ (two-tailed). Descriptive statistics were calculated and depicted as percentage, mean and standard deviation or median and range. Data distribution was evaluated using Shapiro-Wilk and Kolmogorov-Smirnov tests. As not all data were distributed normally and considering the small number of participants, we chose non-parametric statistics. A Mann-Whitney $U$ test for independent samples was used to determine differences between groups of dichotomous variables. All data were checked for outliers and, if present, analyses were repeated without them. Correlation was studied by means of Spearman rank correlation and, if suitable, linear regression was used to model the relationship between two metric variables. To evaluate outcome measures longitudinally, a two-way analysis of variance by ranks (Friedman's test) for dependent samples was applied.

This study report was structured following the reporting guidelines to strengthening the Reporting of Observational Studies in Epidemiology (STROBE) [43].

\section{Results}

\section{Participant characteristics}

18 patients with SMA type II $(N=6)$, IIIa $(N=4), \operatorname{IIIb}(N=7)$ and IV $(N=1)$ were confirmed eligible and enrolled in the study. Ten participants had $\geq 4 S M N 2$ copies, whereof two participants reported symptom onset within 36 months after birth. Participant characteristics are summarized in Table 1. 
Table 1 Socio-demographic and disease characteristics at baseline

\begin{tabular}{|c|c|c|c|}
\hline & $N(\%)$ & Mean (SD) & Median (Range) \\
\hline Female gender & 7 (38.9) & & \\
\hline Age at therapy start (y) & 18 & $37.1(13.1)$ & $34(19-64)$ \\
\hline Symptom duration (y) & 18 & $28.6(13.6)$ & $30(2-50)$ \\
\hline \multicolumn{4}{|l|}{ SMA type } \\
\hline Type II & $6(33.3)$ & & \\
\hline Type IIIa & $4(22.2)$ & & \\
\hline Type IIIb & 7 (38.9) & & \\
\hline Type IV & $1(5.6)$ & & \\
\hline \multicolumn{4}{|l|}{$S M N 2$ copy number } \\
\hline$<4$ & $8(44.4)$ & & \\
\hline$\geq 4$ & $10(55.6)$ & & \\
\hline \multicolumn{4}{|l|}{ Walking ability } \\
\hline Non-ambulatory & $9(50.0)$ & & \\
\hline Ambulatory & $9(50.0)$ & & \\
\hline Scoliosis & 7 (38.9) & & \\
\hline NIV & $4(22.2)$ & & \\
\hline PEG & $1(5.6)$ & & \\
\hline $\mathrm{BMI}<18.5$ & $5(27.8)$ & & \\
\hline Education $>12$ years & $15(83.3)$ & & \\
\hline Living with a partner & $7(38.9)$ & & \\
\hline 6MWT distance $(\mathrm{m})$ & 9 & $345.4(169)$ & $415(42-512)$ \\
\hline HFMSE & 18 & $27.2(25.3)$ & $19.5(0-64)$ \\
\hline RULM & 18 & $23.4(11.8)$ & $23.5(0-37)$ \\
\hline
\end{tabular}

Depicted are participant and disease characteristics as number (percentage), mean with standard deviation or median with range. The cut-off value for the education level was determined at 12 years as school attendance in most countries lasts up to 12 years. Remarkably, this sample displayed a high educational level with $83.3 \%$ reporting more than 12 years of formal education. Previous studies reported similar education levels in adult SMA patients [57]

SMA spinal muscular atrophy, SMN2 survival of motor neuron 2 gene, NIV non-invasive ventilation, $P E G$ percutaneous endoscopic gastrostomy, $B M I$ body mass index, $y$ years, $6 M W T$ 6-Minutes Walk Test, $m$ meter, HFMSE Hammersmith Functional Motor Scale Expanded, $R U L M$ Revised Upper Limb Module, $N$ number, $S D$ standard deviation

Mean age at first nusinersen administration was 37.1 years, mean duration of symptoms was 28.6 years. Half of the participants were ambulatory $(N=9)$, whereby ambulatory was defined as being able to walk without support for at least 10 meters [44]. Seven participants had scoliosis, four were dependent on intermittent non-invasive ventilation (NIV) and one had percutaneous endoscopic gastrostomy (PEG). Taken together, the study sample was severely impaired as reflected by mean HFMSE (27.2/66) and RULM (23.4/37).

\section{Prevalence and dimensions of fatigue}

At baseline, 53\% of participants were classified as abnormally fatigued according to the FSS (FSS $>4$ ), thereof six participants reported severe fatigue (FSS $>5$ ). 53\% agreed that fatigue was among their three most disabling symptoms according to item 8 of the FSS (item $8 \geq 5$ ). Considering MFI, age- and sex-adjusted prevalence of general fatigue was $75 \%$ in the total sample and all but one participant (94\%) reported abnormal physical fatigue. Detailed prevalence rates and fatigue scores at baseline are shown in Table 2.

\section{Factors associated with fatigue}

No significant differences between mean FSS or MFI scores were found regarding gender, living with a partner (yes versus (vs.) no), SMA type (types II/IIIa and types IIIb/IV were grouped together due to small sample sizes), scoliosis (yes vs. no), NIV (yes vs. no), BMI ( $<18.5$ vs. $\geq 18.5$ ) and presence of pain/discomfort (EQ-5D-5L pain/discomfort dimension level one vs. levels two to five).

Participants with $\geq 4 S M N 2$ copies (and thus less severely affected) scored significantly higher in the MFI reduced activity domain compared to participants with less than four copies $(p=0.009)$. Similarly, ambulatory participants reported significantly higher levels of fatigue in the MFI reduced activity domain compared to non-ambulatory participants $(p=0.005)$ (Fig. 1a, b).

Regarding anxiety/depression (level one in the EQ5D-5L anxiety/depression domain vs. levels two to five), significant differences were found for mental fatigue: participants without anxiety/depression reported less mental fatigue ( $p=0.023$ ) compared to those who had slight to extreme anxiety/depression. Further, participants with higher education levels ( $>12$ years of school attendance) reported significantly lower general fatigue with the limitation that in our sample, there were only two participants with $\leq 12$ years of school attendance $(p=0.017)$ (Fig. 1c, d).

Correlation analysis and linear regression of fatigue measures and age at therapy start revealed a significant relationship of the FSS mean score and three MFI subdomains: Participants who were older at the time of treatment initiation reported more general and mental fatigue as well as higher scores on the MFI reduced activity domain (FSS: $p=0.011$, general fatigue: $p=0.007$, mental fatigue: $p=0.014$, reduced activity: $p=0.048$ ) (Fig. 2a-d). Further, a significant positive correlation was observed between symptom duration and mental fatigue $(p=0.019)$ (Fig. 2e). Regarding motor function, we found a positive correlation between RULM scores and the MFI reduced activity domain: participants with better upper limb motor function reported higher fatigue ( $p=0.049)$ (Fig. 2f), whereas HFMSE did not correlate significantly with fatigue measures.

Correlations between FSS and MFI dimensions were as follows: FSS correlated best with general fatigue, but did 
Table 2 Development of fatigue measures and prevalence rates during nusinersen treatment

\begin{tabular}{llllll}
\hline & Baseline $(N=15)$ & Month 2 $(N=17)$ & Month 6 $(N=14)$ & Month 10 $(N=14)$ & Month 14 $(N=14)$ \\
\hline FSS & $4.31(1.51), 53 \%$ & $4.65(1.44), 65 \%$ & $3.93(1.43), 43 \%$ & $4.48(1.35), 64 \%$ & $3.87(1.48), 43 \%$ \\
FSS item 8 & $3.87(2.45), 53 \%$ & $4.53(2.12), 59 \%$ & $3.29(2.20), 43 \%$ & $4.07(1.94), 50 \%$ & $3.86(2.21), 43 \%$ \\
\hline & Baseline $(N=16)$ & Month 2 $(N=17)$ & Month 6 $(N=15)$ & Month $10(N=14)$ & Month $14(N=14)$ \\
\hline General fatigue & $10.69(3.44), 75 \%$ & $10.94(2.97), 82 \%$ & $10.33(2.77), 67 \%$ & $11.46(4.63), 86 \%$ & $9.79(3.97), 57 \%$ \\
Mental fatigue & $8.62(3.54), 56 \%$ & $7.94(3.21), 53 \%$ & $7.93(2.58), 53 \%$ & $8.64(3.84), 57 \%$ & $9.71(3.95), 64 \%$ \\
Physical fatigue & $13.25(4.07), 94 \%$ & $13.24(3.81), 82 \%$ & $11.80(3.99), 73 \%$ & $12.43(2.98), 86 \%$ & $11.36(3.61), 86 \%$ \\
Reduced activity & $8.88(3.14), 56 \%$ & $10.00(3.57), 71 \%$ & $9.20(3.84), 60 \%$ & $10.00(4.02), 50 \%$ & $6.43(2.34), 14 \%$ \\
Reduced motivation & $6.69(2.12), 31 \%$ & $7.18(3.19), 29 \%$ & $6.93(2.58), 7 \%$ & $7.57(3.63), 29 \%$ & $8.00(3.16), 50 \%$ \\
\hline
\end{tabular}

Data are expressed as mean score (SD), percentage of participants with clinically significant fatigue (prevalence). While mean scores of FSS, general fatigue and physical fatigue tended to decrease towards month 14, mental fatigue and reduced motivation slightly increased during treatment

FSS Fatigue Severity Scale, MFI Multidimensional Fatigue Inventory, $S D$ standard deviation, $N$ number

not correlate significantly with physical fatigue or reduced motivation. Mental fatigue correlated significantly with all other scores and general fatigue correlated significantly with all scores but reduced motivation. Physical fatigue did only correlate significantly with general and mental fatigue, but not with reduced activity or reduced motivation (data not shown).

\section{Factors associated with health-related quality of life (HRQOL)}

HRQOL represented by EQ-5D-5L index values was not normally distributed in our sample (mean $=0.46, \mathrm{SD}=0.37$; median $=0.18$, range $=0.03-1.00)$, but showed a cluster formation: one cluster with an index value below $0.2(N=9)$ and another cluster with an index value above $0.68(N=7)$. Accordingly, we presumed that index values in our cohort were not representative for HRQOL in 5q-SMA and, thus, excluded the index values from further analysis. In contrast, EQ-5D-5L VAS values were distributed normally with a mean value of $57.06(\mathrm{SD}=19.61)$ and median value of 60 (range 25-100).

No significant differences in HRQOL measured by EQ5D-5L VAS could be detected for the variables gender, BMI, education level, relationship status, prevalence of pain/discomfort or depression/anxiety, SMA type, SMN2 copy number, scoliosis, walking ability, NIV or age at therapy onset. Correlation analysis, on the other hand, revealed a negative relation of HRQOL with symptom duration $(p=0.039)$ and a positive relation with HFMSE and RULM scores $(p=0.032$ and $p=0.04$ ) (Fig. 3a-c). Further, correlation analysis of fatigue measures and EQ-5D-5L VAS showed a significant linear relationship for physical fatigue $(p=0.012)$ : participants who reported higher physical fatigue scores presented with lower health-related quality of life (Fig. 3d).

\section{Development of perceived fatigue during nusinersen treatment}

By applying Friedman's test, no significant differences between fatigue scores at different times during nusinersen treatment were detected (Table 2). Mean scores for item 8 of the FSS decreased during treatment ( $p=0.026$, test statistic $=11.038$, degrees of freedom $=4, N=12$ ), but pairwise comparison did not reveal any significant changes. There was a trend towards a decrease in fatigue during treatment, with the lowest score after 14 months for FSS, general and physical fatigue. Mean scores for reduced activity also decreased towards month $14(p=0.024$, test statistic $=11.262$, degrees of freedom $=4, N=12$ ), but significance was also slightly missed after Bonferroni correction in pairwise comparison. Mental fatigue and reduced motivation in contrast showed a tendency to increase towards month 14 (Fig. 4a) (Table 2). In subgroup analysis, participants with $\geq 4$ SMN2 copies and ambulatory participants showed a significant decline in the reduced activity domain from baseline to month 14 $(p=0.026)$ (Fig. 4b).

As mean scores represent raw data and prevalence rates via MFI were adjusted for age and gender, we additionally evaluated the change of prevalence rates. The prevalence of physical fatigue and reduced motivation showed a tendency to decrease during nusinersen treatment with lowest scores at month 6. After month 6, reduced motivation tended to increase along with mental fatigue. The prevalence of general fatigue fluctuated and prevalence of reduced activity increased within the first two treatment months followed by a remarkable decrease afterwards. According to FSS item 8 ("Fatigue is among my three most disabling symptoms"), fatigue was a relevant complaint in $43 \%$ of the participants at month 14 , compared to $53 \%$ at baseline (Table 2). 


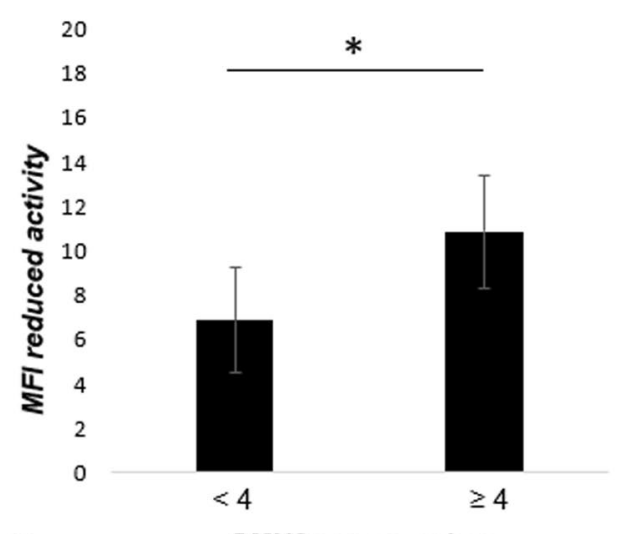

a

SMN2 copy number

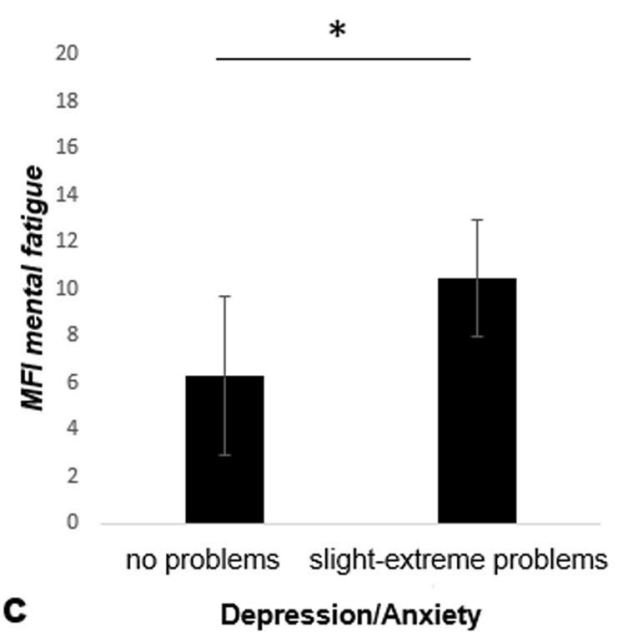

Fig. 1 Association of fatigue with socio-demographic and disease characteristics. Mean score and standard deviation for each group are shown, ${ }^{*} p<0.05$. a Higher scores in the reduced activity domain were reported by participants with $\geq 4 S M N 2$ copies: $\geq 4$ copies, mean $=10.88, \mathrm{SD}=2.53, N=8 ;<4$ copies, mean $=6.88, \mathrm{SD}=2.36$, $N=8(p=0.009, Z=-2.545$, Mann-Whitney $U=8.000)$. b Ambulatory participants reported higher scores in the reduced activity domain: ambulatory, mean $=10.88, \mathrm{SD}=2.53, N=8$; non-ambulatory, mean $=6.88, \mathrm{SD}=2.36, N=8(p=0.005, Z=-2.672$, Mann-

\section{Fatigue compared to motor function development}

To evaluate the suitability of fatigue as an additional outcome measure, we further investigated fatigue scores compared to the development of motor function under nusinersen therapy. Participants were dichotomized into two groups: participants whose RULM/HFMSE scores increased during 14 months of treatment were compared to participants with deteriorated or unchanged RULM/ HFMSE scores, as an improvement of motor functions is not observed in the natural course of SMA and most likely is a therapy effect. Mean changes in RULM scores for these subgroups were $+3(\mathrm{SD}=2, N=3)$ and \pm 0
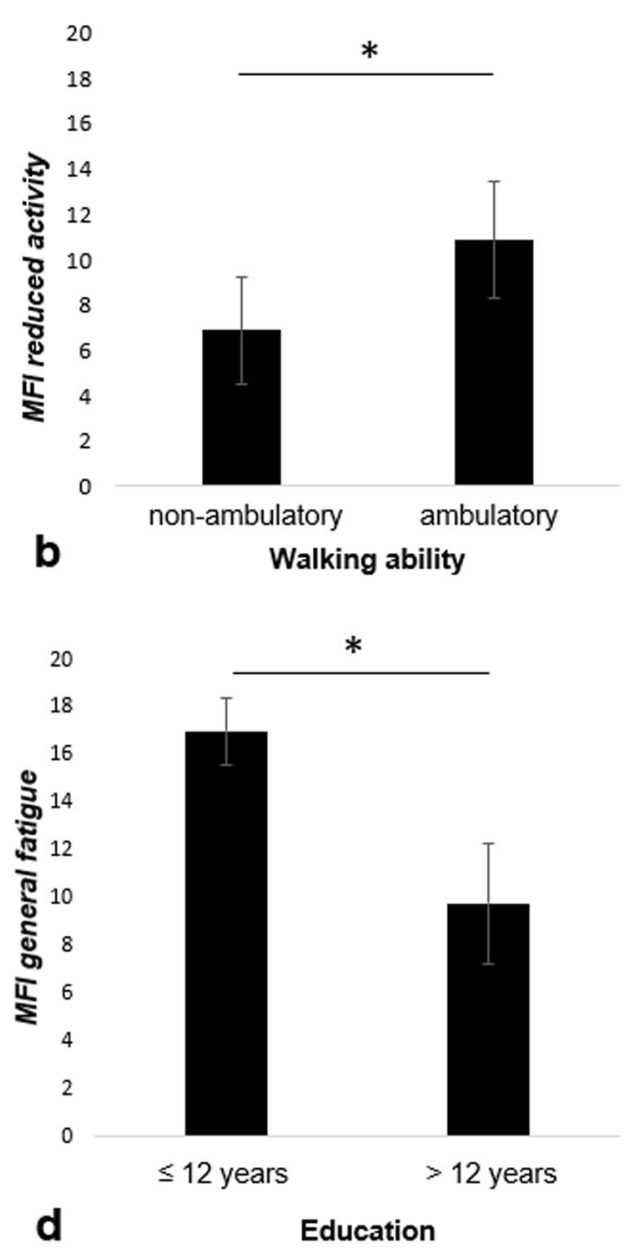

Whitney $U=6.500)$. c Higher scores in mental fatigue were reported by anxious or depressed participants: no problems with anxiety/ depression, mean $=6.29, \mathrm{SD}=3.40, N=7$; slight—extreme problems with anxiety/depression, mean $=10.44, \mathrm{SD}=2.51, N=9(p=0.023$, $Z=-2.305$, Mann-Whitney $U=10.000$ ). d Participants with twelve or less years of formal education reported higher general fatigue scores: $\leq 12$ years, mean $=17.00, \mathrm{SD}=1.414, N=2 ;>12$ years, mean $=9.79, \mathrm{SD}=2.547, N=14 \quad(p=0.017, Z=-2.239$, MannWhitney $U=0.000$ )

$(N=11)$. None of the participants showed a decrease of RULM score. Initial fatigue scores at baseline did not differ significantly, but there was a trend that participants who showed a RULM score increase reported less fatigue, especially less physical fatigue. At months 2, 6 and 10, this trend became statistically significant for physical fatigue (month 2: $p=0.028, N=13, \mathrm{Z}=-2.133$, Mann-Whitney $U=2.500$; month 6: $p=0.014, N=13, Z=-2.35$, Mann-Whitney $U=1.500$; month 10: $p=0.036, N=12$, $Z=-2.16$, Mann-Whitney $U=2.000$ ). Mental fatigue was reported significantly less by participants with improved RULM scores at month $2(p=0.007, N=13, Z=-2.58$, Mann-Whitney $U=0.000)$ and month $6(p=0.007$, 

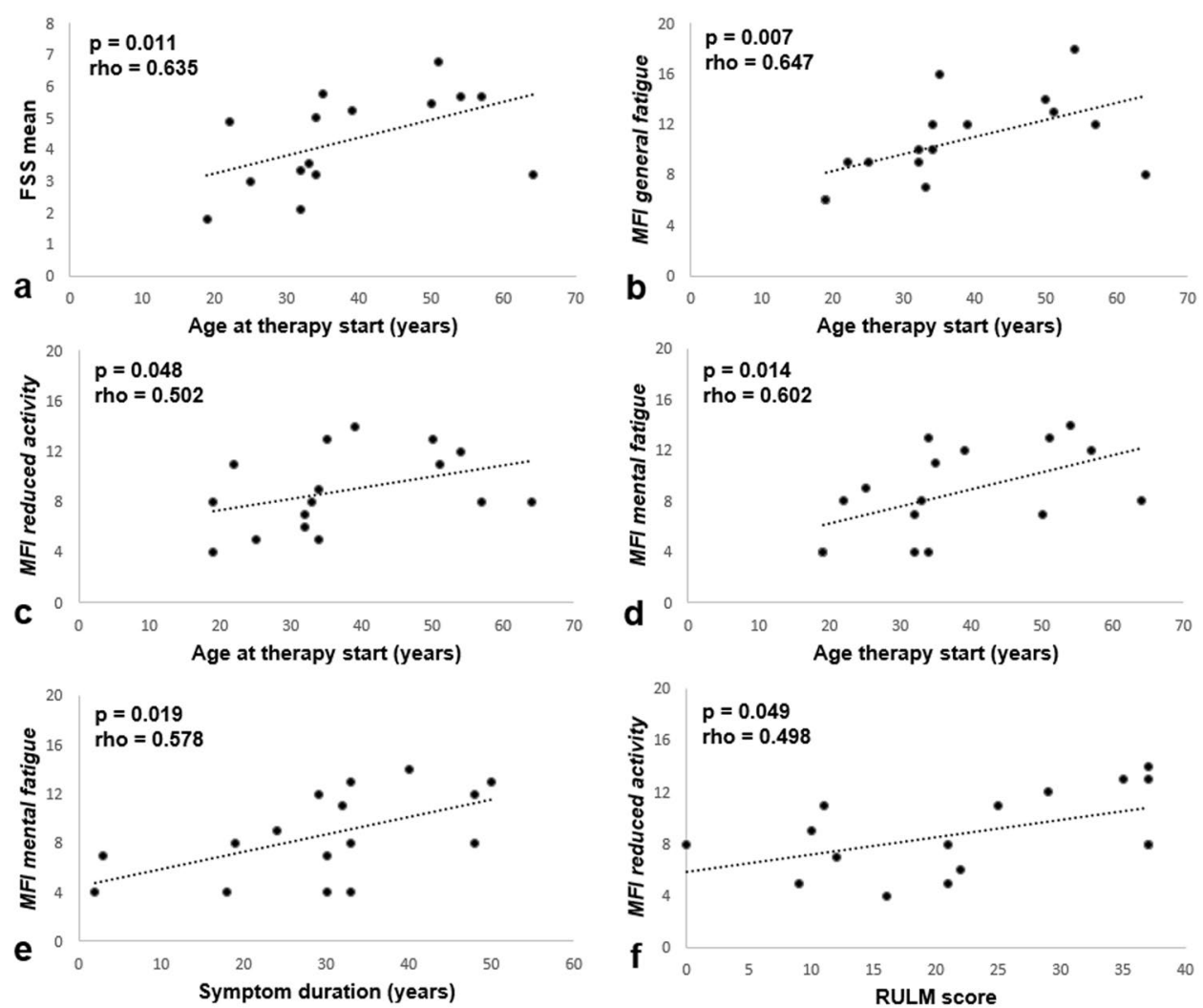

Fig. 2 Correlation of fatigue measures, age at therapy start, symptom duration and motor function. Adjustment curves of linear regression analyzes are shown. Each dot represents one participant. $\mathbf{a}+\mathbf{b}$ Fatigue Severity Scale (FSS) mean score (Spearman-Rho $=0.635$, $\left.p=0.011, R^{2}=0.251, N=15\right)$ and general fatigue assessed via Multidimensional Fatigue Inventory (MFI) (Spearman-Rho $=0.647$, $p=0.007, R^{2}=0.289, N=16$ ) correlated positively with age at therapy start. $\mathbf{c}$ The reduced activity domain of the MFI correlated posi-

$N=13, Z=-2.62$, Mann-Whitney $U=0.000$ ). At month 14 , no significant differences between RULM groups were observed. However, these findings have to be interpreted with care due to the small number of participants in the group with improved RULM score $(N=3)$. Mean changes in HFMSE scores were $+3.13(\mathrm{SD}=4.05, N=8)$ and $-1.43(\mathrm{SD}=1.40, N=7)$, accordingly. There were no significant differences in fatigue scores between participants with increased HFMSE during treatment and those with deteriorated/stable HFMSE at any time.

\section{Discussion}

In this study, adult 5q-SMA patients frequently reported fatigue as one of their most disabling symptoms. Physical fatigue followed by general fatigue and reduced activity were identified as the most relevant dimensions. We

tively with age at therapy start (Spearman-Rho $=0.502, p=0.048$, $\left.R^{2}=0.151, N=16\right)$. $\mathbf{d}+\mathbf{e}$ Mental fatigue correlated positively with age at therapy start (Spearman-Rho $=0.602, p=0.014, R^{2}=0.281$, $N=16)$ and symptom duration (Spearmen-Rho $=0.578, p=0.019$, $\left.R^{2}=0.319, N=18\right)$. f Participants with higher RULM scores reported more fatigue in the reduced activity domain of the MFI (SpearmanRho $=0.498, p=0.049, R^{2}=0.264, N=16$ )

found that socio-demographic factors were associated with general and mental fatigue, while disease characteristics and motor function were associated with reduced activity. Physical fatigue correlated negatively with HRQOL, but showed no association with participant or disease characteristics. Interestingly, after more than one year of nusinersen treatment, less participants tended to report fatigue as one of their most disabling symptoms and there was a trend towards a decrease of physical and general fatigue as well as reduced activity. In the subgroup of ambulatory participants, reduced activity significantly improved at month 14. Participants with increasing RULM scores under therapy reported significantly less physical fatigue between month 2 and 10 of treatment as well less mental fatigue between month 2 and 6 compared to participants with unchanged RULM scores.

To measure multiple dimensions of fatigue in our study cohort, we made use of two independent questionnaires. 

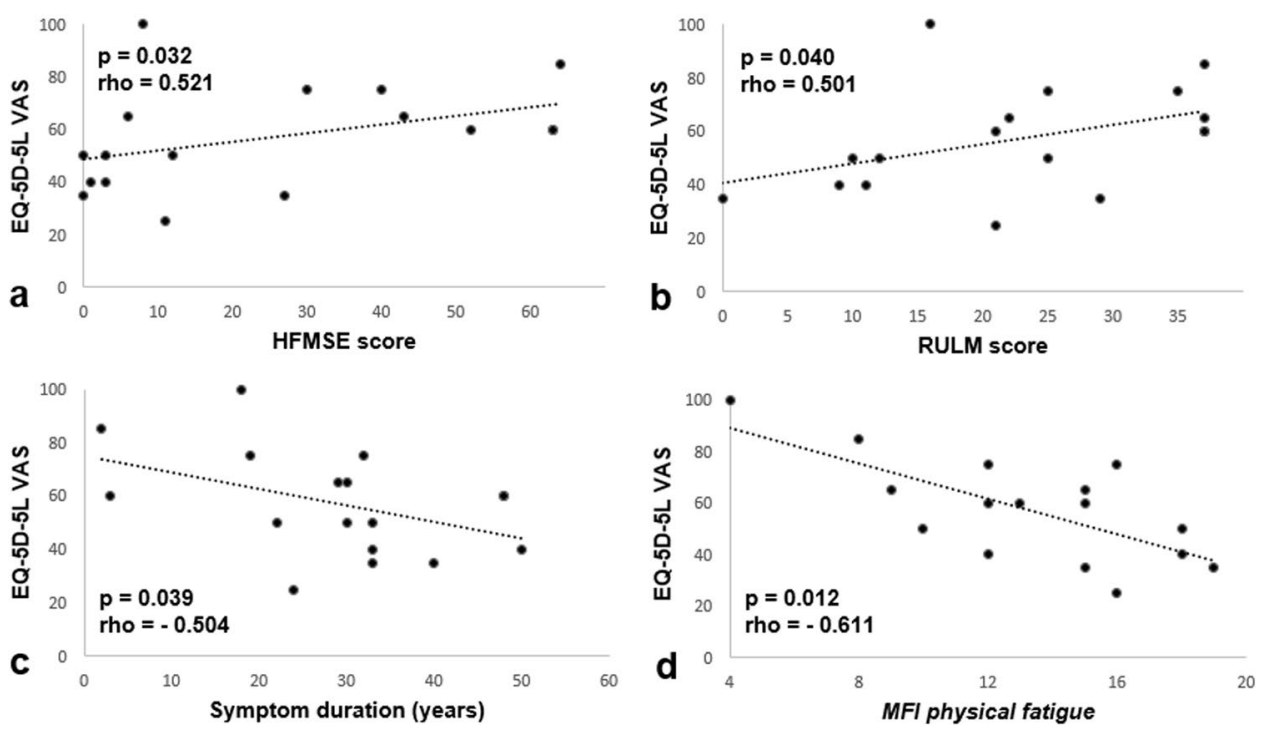

Fig. 3 Factors associated with health-related quality of life (HRQOL). Adjustment curves of linear regression analyzes are shown. Each dot represents one participant. a-c HRQOL via EQ-5D-5L VAS correlated positively with HFMSE (SpearmanRho $=0.521, p=0.032, R^{2}=0.172, N=17$ ) and RULM scores (Spearman-Rho $\left.=0.501, p=0.040, N=17, R^{2}=0.181\right)$. We further observed
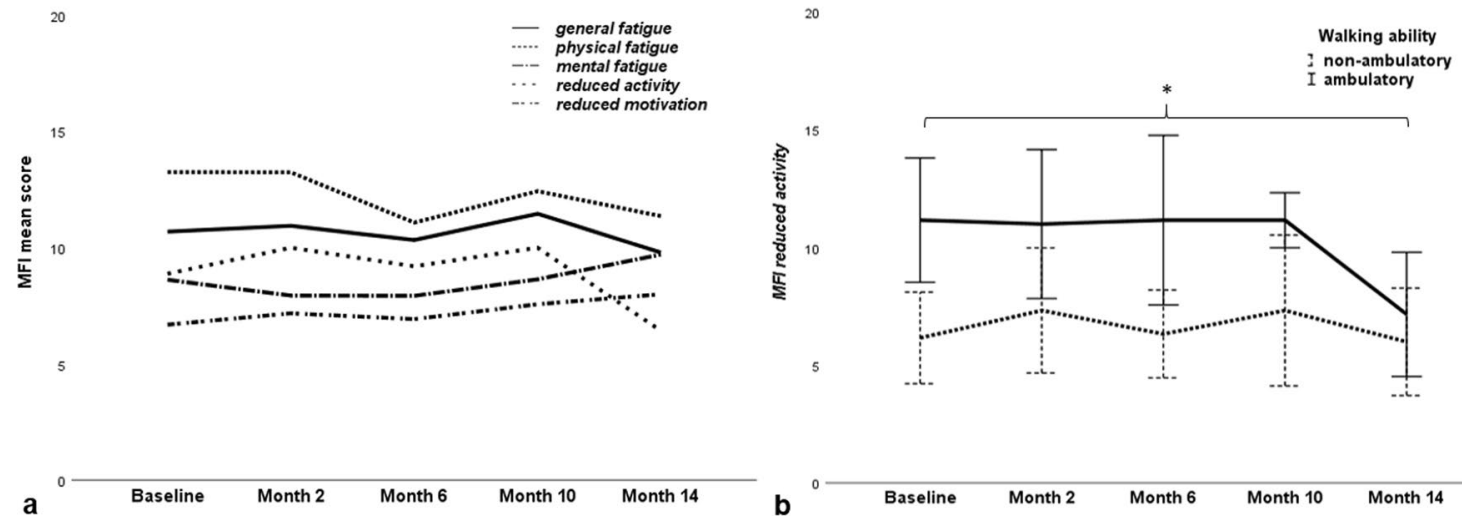

a negative correlation of HRQOL via EQ-5D-5L VAS with symptom duration (Spearman-Rho $=-0.504, p=0.039, R^{2}=0.191, N=17$ ). d The adjustment curve of linear regression analysis of HRQOL and physical fatigue assessed via Multidimensional Fatigue Inventory (MFI) showed a significant negative correlation (SpearmanRho $=-0.611, p=0.012, R^{2}=0.487, N=16$ )

\section{b}

\begin{tabular}{ccccc}
\hline Baseline & Month 2 & Month 6 & Month 10 & Month 14 \\
\hline
\end{tabular}

Fig. 4 Development of fatigue under nusinersen treatment. a Mean scores of the five MFI dimensions are depicted during 14 months of nusinersen treatment. While general fatigue, physical fatigue and reduced activity tended to decline, mental fatigue and reduced motivation showed a tendency to increase towards month 14 . b Mean scores and standard deviations of reduced activity are depicted dur-

ing nusinersen treatment. The dashed line represents the non-ambulatory participants, the solid line the ambulatory participants. There was a significant decline of reduced activity in ambulatory participants at month $14[p=0.018, N=6$, test statistic $=11.930$, degrees of freedom $=4, p$ (pairwise comparison between baseline and month 14) $=0.026], * p<0.05$

While the FSS has been validated in SMA patients [20], this is - to our knowledge - the first study involving the MFI. The mean FSS score at baseline was 4.31 which is consistent with the recent report of Kizina et al. who found a mean score of 4.61 in adult SMA patients awaiting nusinersen treatment [45]. The discrepancy between the prevalence rates of 53\% (FSS) and 75\% (MFI general fatigue) might be explained by the nature of the assessment instruments: while the FSS measures the impact of fatigue on functioning, the MFI primarily measures phenomenology and severity of fatigue. Further and most importantly, prevalence was reported as raw data for the FSS. Meanwhile, for MFI, ageand gender-adjusted cut-off values were used as suggested by Singer et al. [32]. As fatigue is dependent on age and gender [46, 47], which in part was also applicable in our cohort, prevalence estimation might be more accurate using those age- and gender-adjusted cut-off values and the estimated prevalence of $75 \%$ via MFI seems to be more precise. This 
is in line with the previously reported prevalence of fatigue of $81-100 \%$ in spinobulbar muscular atrophy (SBMA) and SMA patients assessed via FSS and PedsQL ${ }^{\text {TM }}$ Multidimensional Fatigue Scale [24, 25]. Regarding the development in fatigue over the course of 14 months, both instruments depict a similar relative change for general fatigue, which indicates their suitability to measure the same construct in the long term.

In the present study, $94 \%$ of the participants reported abnormal physical fatigue, which indicated its importance in adult SMA. As patients with disabling motor neuron diseases are not able to perform exercise in the classical sense (e.g. running or weight lifting), they tend to report less activity determined fatigue [48], which is assessed by most fatigue instruments (for example FSS item 2: "Exercise brings on my fatigue"). The MFI, on the other hand, addresses the perceived physical condition in general rather than fatigue following exercise and might, therefore, be more sensitive in SMA patients (for example item 2: "Physically I feel only able to do a little" or item 14: "Physically I feel I am in a bad condition"). In accordance with previous reports regarding fatigue in general [14, 21, 25], physical fatigue did not correlate with disease severity or motor function in our cohort. Nevertheless, it was associated with deteriorated HRQOL as previously described for fatigue in motor neuron diseases [49], while HRQOL itself correlated positively with measures of motor function. Further, physical fatigue showed a tendency to decrease under treatment and was temporarily significantly lower in the subgroup of participants with improved RULM scores. Therefore, physical fatigue appears to be a symptom independent of motor function which has impact on HRQOL and a further evaluation as PRO seems worthwhile. In contrast, the FSS did not correlate with HRQOL or resemble motor improvements in our cohort and seems to be less suitable as outcome measure in adult SMA patients in the short term.

Next, we found that older participants and participants with $<12$ years of education suffered more extensively from general fatigue according to FSS and MFI, which is in line with findings in the general population and might be an effect independent of SMA [46, 47]. Though, the correlation between education and fatigue should be interpreted with care regarding the size of our cohort $(\leq 12$ years of education: $N=2$ ). Mental fatigue scores were higher in participants with depression/anxiety, longer symptom duration and higher age at therapy start. These effects have also been shown for general and mental fatigue in other neurological diseases, such as multiple sclerosis and Parkinson's disease $[15,50,51]$. Surprisingly, we found no effect of discomfort/ pain on perceived fatigue as it has been shown for various conditions $[33,52,53]$. A possible explanation might be that pain is not a primary symptom in SMA. Reduced motivation seemed to be less of a problem in SMA patients with a mean score of 6.69 , which is comparable to general population [54].

In contrast to the other fatigue dimensions, subgroup and correlation analysis of reduced activity revealed significantly higher scores in less severely impaired participants $(\geq 4$ SMN2 copies, ambulatory, higher RULM scores) and after 14 months of nusinersen treatment reduced activity significantly improved within this subgroup. Nonetheless, especially the domain of reduced activity might be sensitive to SMA severity. Patients with a higher SMN2 copy number might experience a greater mismatch between their own reached best activity level (before disease onset) and their actual capacities/activities compared to those with less $S M N 2$ copies who, e.g. never learned to walk. Additionally, patients with milder disease severity and later disease onset might be less adapted to their impairment (fewer auxiliary devices and personnel) and, therefore, experience more limitations in their activities. We assume that reduced activity might not reflect perceived fatigue in our cohort but rather a phenomenon independent of fatigue as it is associated with disease severity but not with quality of life.

Recently, fatigability in ambulatory children and adolescent SMA patients (measured by physiotherapists as distance walked in the 6MWT) has shown clinically meaningful improvement under nusinersen treatment [55]. As opposed to this study, we used assessments of perceived fatigue feasible for all patients independent of motor function impairment. Overall, the most prevalent fatigue dimensions tended to decrease during 14 months of nusinersen treatment. As there are no data available on the natural course of fatigue among SMA patients, the interpretation of our results remains difficult. However, fewer participants tended to rate fatigue as one of their most disabling symptoms at month 14. Reduced activity showed a tendency to increase intermittently during treatment with nusinersen and reached its maximum at month 2 . This might reflect the increased treatment burden during the loading period. During the maintenance period, reduced activity levels remarkably declined. Temporary improvements in mental fatigue and reduced motivation, on the other hand, might have been biased by positive pre-treatment expectations, while increased scores after ten and 14 months could reflect treatment burden or reluctance. Prevalence of general fatigue (via MFI and FSS) tended to be decreased at month 14 compared to baseline. In general, we observed a fluctuation of fatigue scores and prevalence rates in the longitudinal assessment. This underlines the exploratory character of our study. An investigation over a longer time period seems to be unavoidable to detect relevant treatment effects because of the slowly progressive disease course in the investigated patient cohort. Longer observation and treatment duration are also needed to assess long-term efficacy and sustainability of effects. 
This study has further limitations. Primarily, it is limited by the relatively small sample size of 18 participants due to which we might not have been able to demonstrate significant effects of covariates or treatment. To improve generalizability, analyses of larger samples including SMA type I patients should be initiated through national and international cooperation. Due to the absence of a control group, we can only calculate absolute effect measures. However, withholding nusinersen treatment from SMA patients is ethically not justifiable and there seem to be only few SMA patients who decline nusinersen treatment. Further, not all conceivable covariates which might influence perceived fatigue, such as sleep disturbances, dyspnea, hemoglobin levels or employment status, were recorded $[33,56]$. Finally, we have to take the possibility of selection bias into account as we cannot be totally sure that the participating SMA patients are representative of all SMA patients. Patients with longer symptom duration might not have come forward to seek treatment, which might have led to an underestimation of fatigue prevalence.

In conclusion, we characterized perceived fatigue in adult SMA patients and identified it as a frequent and burdening symptom. While disease severity and motor function were associated with reduced activity, socio-demographic factors as age, education and depression influenced general and mental fatigue. Physical fatigue as most prevalent dimension of fatigue correlated negatively with HRQOL, but was independent of participant and disease characteristics. Fatigue measures fluctuated during 14 months of nusinersen treatment with a trend towards reduced physical and general fatigue and improved reduced activity. Perceived fatigue, especially physical fatigue and reduced activity, appears to be a suitable PRO additionally to motor function outcome measures, as it (1) is frequent in SMA, (2) is a complaint relevant to patients which correlates with quality of life, (3) can be assessed easily and (4) might be more sensitive in detecting subjective improvement/worsening in more severely affected patients than motor function measures. Larger patient cohorts and longer observation periods are needed to further address this suggestion.

Acknowledgements The authors wish to thank all participants and their caregivers for supporting this study; as well as the therapists who routinely assessed motor function (Department of Rehabilitation Medicine, Hannover Medical School).

Author contributions $\mathrm{CB}$ performed data collection, analysis and interpretation and wrote the first draft of the manuscript. OS-K was involved in study planning, data collection and interpretation and critically reviewed the manuscript. MK, GR, HS and GW collected data and critically reviewed the manuscript. SP planned the study, was involved in coordination of the study, was responsible for local data collection and contribution, and critically revised the manuscript. AO planned and coordinated the study, performed data collection and interpretation, and supported writing of the manuscript. All authors read and approved the final version of the manuscript.

Funding Open Access funding enabled and organized by Projekt DEAL. No targeted study funding. Olivia Schreiber-Katz is receiving academic research support from the Hannover Medical School Young Faculty Program, 2018-2020. Susanne Petri has received grants from the German Neuromuscular Society, Federal Ministry of Education and Research, German Israeli Foundation for Scientific Research and Development, EU Joint Programme for Neurodegenerative Disease Research. Alma Osmanovic is being supported by PRACTISClinician Scientist Program of Hannover Medical School, funded by the German Research Foundation (DFG, ME 3696/3-1, 2020-2022).

Availability of data and material De-identified data will be shared on reasonable request with any qualified investigator

\section{Compliance with ethical standards}

Conflicts of interest Camilla Binz reports no disclosures. Olivia Schreiber-Katz has received honoraria as a speaker/consultant and/or funding for travel expenses from the German Neuromuscular Society "Deutsche Gesellschaft fuer Muskelkranke (DGM e.V.), Novartis, Biogen GmbH, Biermann Verlag GmbH and the Jain Foundation. She has received research support from the German Neuromuscular Society "Deutsche Gesellschaft fuer Muskelkranke (DGM e.V.), 2019-2020. Mareike Kumpe and Gresa Ranxha received travel cost compensation from Biogen $\mathrm{GmbH}$. Hannah Siegler and Gary Wieselmann report no disclosures. Susanne Petri has received speaker/ consultant honoraria and funding for travel expenses from Biogen, Cytokinetics, Inc., Desitin Pharma, Novartis, Roche, Teva. Alma Osmanovic has received honoraria as a speaker/consultant from the German Neuromuscular Society "Deutsche Gesellschaft fuer Muskelkranke (DGM e.V.) and Biogen GmbH.

Ethics approval The study has been approved by the local ethics review board of Hannover Medical School (no. 6269) and has been performed in accordance with the ethical standards laid down in the 1964 Declaration of Helsinki and its later amendments.

Consent to participate All patients gave their written informed consent before entering the study.

Consent for publication All study participants gave their written informed consent to the publication of anonymized cohort data.

Open Access This article is licensed under a Creative Commons Attribution 4.0 International License, which permits use, sharing, adaptation, distribution and reproduction in any medium or format, as long as you give appropriate credit to the original author(s) and the source, provide a link to the Creative Commons licence, and indicate if changes were made. The images or other third party material in this article are included in the article's Creative Commons licence, unless indicated otherwise in a credit line to the material. If material is not included in the article's Creative Commons licence and your intended use is not permitted by statutory regulation or exceeds the permitted use, you will need to obtain permission directly from the copyright holder. To view a copy of this licence, visit http://creativecommons .org/licenses/by/4.0/. 


\section{References}

1. Lefebvre S, Burglen L, Reboullet S, Clermont O, Burlet P, Viollet L, Benichou B, Cruaud C, Millasseau P, Zeviani M (1995) Identification and characterization of a spinal muscular atrophydetermining gene. Cell 80:155-165. https://doi.org/10.1016/00928674(95)90460-3

2. Kolb SJ, Kissel JT (2015) Spinal muscular atrophy. Neurol Clin 33:831-846. https://doi.org/10.1016/j.ncl.2015.07.004

3. Wirth B, Brichta L, Schrank B, Lochmuller H, Blick S, Baasner A, Heller R (2006) Mildly affected patients with spinal muscular atrophy are partially protected by an increased SMN2 copy number. Hum Genet 119:422-428. https://doi.org/10.1007/s0043 9-006-0156-7

4. Finkel RS, Mercuri E, Darras BT, Connolly AM, Kuntz NL, Kirschner J, Chiriboga CA, Saito K, Servais L, Tizzano E, Topaloglu H, Tulinius M, Montes J, Glanzman AM, Bishop K, Zhong ZJ, Gheuens S, Bennett CF, Schneider E, Farwell W, De Vivo DC, ENDEAR Study Group (2017) Nusinersen versus sham control in infantile-onset spinal muscular atrophy. N Engl J Med 377:17231732. https://doi.org/10.1056/NEJMoa1702752

5. Mercuri E, Darras BT, Chiriboga CA, Day JW, Campbell C, Connolly AM, Iannaccone ST, Kirschner J, Kuntz NL, Saito K, Shieh PB, Tulinius M, Mazzone ES, Montes J, Bishop KM, Yang Q, Foster R, Gheuens S, Bennett CF, Farwell W, Schneider E, De Vivo DC, Finkel RS, CHERISH Study Group (2018) Nusinersen versus sham control in later-onset spinal muscular atrophy. $\mathrm{N}$ Engl J Med 378:625-635. https://doi.org/10.1056/NEJMoa1710504

6. Veerapandiyan A, Eichinger K, Guntrum D, Kwon J, Baker L, Collins E, Ciafaloni E (2019) Nusinersen for older patients with spinal muscular atrophy: a real-world clinical setting experience. Muscle Nerve. https://doi.org/10.1002/mus.26769

7. Cordts I, Lingor P, Friedrich B, Pernpeintner V, Zimmer C, Deschauer M, Maegerlein C (2020) Intrathecal nusinersen administration in adult spinal muscular atrophy patients with complex spinal anatomy. Ther Adv Neurol Disord 13:1756286419887616. https://doi.org/10.1177/1756286419887616

8. Walter MC, Wenninger S, Thiele S, Stauber J, Hiebeler M, Greckl E, Stahl K, Pechmann A, Lochmuller H, Kirschner J, Schoser B (2019) Safety and treatment effects of nusinersen in longstanding adult 5q-SMA type 3-a prospective observational study. J Neuromuscul Dis 6:453-465. https://doi.org/10.3233/JND-190416

9. Hagenacker T, Hermann A, Kamm C, Walter MC, Weiler M, Gunther R, Wurster CD, Kleinschnitz C (2019) Spinal muscular atrophy-expert recommendations for the use of nusinersen in adult patients. Fortschr Neurol Psychiatr 87:703-710. https://doi. org/10.1055/a-0996-0994

10. Hagenacker T, Wurster CD, Gunther R, Schreiber-Katz O, Osmanovic A, Petri S, Weiler M, Ziegler A, Kuttler J, Koch JC, Schneider I, Wunderlich G, Schloss N, Lehmann HC, Cordts I, Deschauer M, Lingor P, Kamm C, Stolte B, Pietruck L, Totzeck A, Kizina K, Monninghoff C, von Velsen O, Ose C, Reichmann H, Forsting M, Pechmann A, Kirschner J, Ludolph AC, Hermann A, Kleinschnitz C (2020) Nusinersen in adults with 5q spinal muscular atrophy: a non-interventional, multicentre, observational cohort study. Lancet Neurol 19:317-325. https://doi.org/10.1016/ S1474-4422(20)30037-5

11. Osmanovic A, Ranxha G, Kumpe M, Muschen L, Binz C, Wiehler F, Paracka L, Korner S, Kollewe K, Petri S, Schreiber-Katz O (2020) Treatment expectations and patient-reported outcomes of nusinersen therapy in adult spinal muscular atrophy. J Neurol. https://doi.org/10.1007/s00415-020-09847-8

12. Kaufmann P, McDermott MP, Darras BT, Finkel RS, Sproule DM, Kang PB, Oskoui M, Constantinescu A, Gooch CL, Foley AR, Yang ML, Tawil R, Chung WK, Martens WB, Montes J, Battista
V, O’Hagen J, Dunaway S, Flickinger J, Quigley J, Riley S, Glanzman AM, Benton M, Ryan PA, Punyanitya M, Montgomery MJ, Marra J, Koo B, De Vivo DC, Muscle Study Group (MSG), Pediatric Neuromuscular Clinical Research Network for Spinal Muscular Atrophy (PNCR) (2012) Prospective cohort study of spinal muscular atrophy types 2 and 3. Neurology 79:1889-1897. https://doi.org/10.1212/WNL.0b013e318271f7e4

13. Pechmann A, Konig K, Bernert G, Schachtrup K, Schara U, Schorling D, Schwersenz I, Stein S, Tassoni A, Vogt S, Walter MC, Lochmuller H, Kirschner J (2019) SMArtCARE—a platform to collect real-life outcome data of patients with spinal muscular atrophy. Orphanet J Rare Dis. https://doi.org/10.1186/s1302 3-019-0998-4

14. Chaudhuri A, Behan PO (2004) Fatigue in neurological disorders. Lancet 363:978-988. https://doi.org/10.1016/S0140 $-6736(04) 15794-2$

15. Dittner AJ, Wessely SC, Brown RG (2004) The assessment of fatigue: a practical guide for clinicians and researchers. $\mathrm{J}$ Psychosom Res 56:157-170. https://doi.org/10.1016/S0022 -3999(03)00371-4

16. Kluger BM, Krupp LB, Enoka RM (2013) Fatigue and fatigability in neurologic illnesses: proposal for a unified taxonomy. Neurology 80:409-416. https://doi.org/10.1212/WNL.0b013e31827f07b e

17. Tran C, Bril V, Katzberg HD, Barnett C (2018) Fatigue is a relevant outcome in patients with myasthenia gravis. Muscle Nerve 58:197-203. https://doi.org/10.1002/mus.26069

18. Sharma KR, Kent-Braun J, Mynhier MA, Weiner MW, Miller RG (1994) Excessive muscular fatigue in the postpoliomyelitis syndrome. Neurology 44:642-646. https://doi.org/10.1212/ wnl.44.4.642

19. Tersteeg IM, Koopman FS, Stolwijk-Swuste JM, Beelen A, Nollet F, CARPA Study Group (2011) A 5-year longitudinal study of fatigue in patients with late-onset sequelae of poliomyelitis. Arch Phys Med Rehabil 92:899-904. https://doi.org/10.1016/j. apmr.2011.01.005

20. Werlauff U, Hojberg A, Firla-Holme R, Steffensen BF, Vissing J (2014) Fatigue in patients with spinal muscular atrophy type II and congenital myopathies: evaluation of the fatigue severity scale. Qual Life Res 23:1479-1488. https://doi.org/10.1007/s1113 6-013-0565-8

21. Ramirez C, Piemonte ME, Callegaro D, Da Silva HC (2008) Fatigue in amyotrophic lateral sclerosis: frequency and associated factors. Amyotroph Lateral Scler 9:75-80. https://doi. org/10.1080/17482960701642502

22. Iannaccone ST, White M, Browne R, Russman B, Buncher R, Samaha FJ (1997) Muscle fatigue in spinal muscular atrophy. J Child Neurol 12:321-326. https://doi.org/10.1177/0883073897 01200507

23. de Groot IJ, de Witte LP (2005) Physical complaints in ageing persons with spinal muscular atrophy. J Rehabil Med 37:258-262. https://doi.org/10.1080/16501970510030156

24. Noto Y, Misawa S, Mori M, Kawaguchi N, Kanai K, Shibuya K, Isose S, Nasu S, Sekiguchi Y, Beppu M, Ohmori S, Nakagawa M, Kuwabara S (2013) Prominent fatigue in spinal muscular atrophy and spinal and bulbar muscular atrophy: evidence of activitydependent conduction block. Clin Neurophysiol 124:1893-1898. https://doi.org/10.1016/j.clinph.2012.12.053

25. Dunaway Young S, Montes J, Kramer SS, Podwika B, Rao AK, De Vivo DC (2019) Perceived fatigue in spinal muscular atrophy: a pilot study. J Neuromuscul Dis 6:109-117. https://doi. org/10.3233/JND-180342

26. Krupp LB, LaRocca NG, Muir-Nash J, Steinberg AD (1989) The fatigue severity scale. Application to patients with multiple sclerosis and systemic lupus erythematosus. Arch Neurol 46:11211123. https://doi.org/10.1001/archneur.1989.00520460115022 
27. de Groot IJ, Voet NB, van Middendorp H, Knoop HJ, Rahbek J, van Engelen BG (2013) 184th ENMC International Workshop: pain and fatigue in neuromuscular disorders: 20-22 May 2011, Naarden, The Netherlands. Neuromuscul Disord 23:1028-1032. https://doi.org/10.1016/j.nmd.2013.06.370

28. Whitehead L (2009) The measurement of fatigue in chronic illness: a systematic review of unidimensional and multidimensional fatigue measures. J Pain Symptom Manag 37:107-128. https://doi. org/10.1016/j.jpainsymman.2007.08.019

29. Anton HA, Miller WC, Townson AF (2008) Measuring fatigue in persons with spinal cord injury. Arch Phys Med Rehabil 89:538542. https://doi.org/10.1016/j.apmr.2007.11.009

30. Fayers PM, Machin D (2015) Quality of life: the assessment, analysis and reporting of patient-reported outcomes, New York

31. Smets EM, Garssen B, Bonke B, De Haes JC (1995) The Multidimensional Fatigue Inventory (MFI) psychometric qualities of an instrument to assess fatigue. J Psychosom Res 39:315-325. https ://doi.org/10.1016/0022-3999(94)00125-O

32. Singer S, Kuhnt S, Zwerenz R, Eckert K, Hofmeister D, Dietz A, Giesinger J, Hauss J, Papsdorf K, Briest S, Brown A (2011) Age- and sex-standardised prevalence rates of fatigue in a large hospital-based sample of cancer patients. Br J Cancer 105:445451. https://doi.org/10.1038/bjc.2011.251

33. Bathen T, Velvin G, Rand-Hendriksen S, Robinson HS (2014) Fatigue in adults with Marfan syndrome, occurrence and associations to pain and other factors. Am J Med Genet A 164A:19311939. https://doi.org/10.1002/ajmg.a.36574

34. Herdman M, Gudex C, Lloyd A, Janssen M, Kind P, Parkin D, Bonsel G, Badia X (2011) Development and preliminary testing of the new five-level version of EQ-5D (EQ-5D-5L). Qual Life Res 20:1727-1736. https://doi.org/10.1007/s11136-011-9903-x

35. EuroQol Group (1990) EuroQol-a new facility for the measurement of health-related quality of life. Health Policy 16:199-208. https://doi.org/10.1016/0168-8510(90)90421-9

36. Ludwig K, Graf von der Schulenburg JM, Greiner W (2018) German value set for the EQ-5D-5L. Pharmacoeconomics 36:663674. https://doi.org/10.1007/s40273-018-0615-8

37. Klug C, Schreiber-Katz O, Thiele S, Schorling E, Zowe J, Reilich P, Walter MC, Nagels KH (2016) Disease burden of spinal muscular atrophy in Germany. Orphanet J Rare Dis. https://doi. org/10.1186/s13023-016-0424-0

38. Lopez-Bastida J, Pena-Longobardo LM, Aranda-Reneo I, Tizzano E, Sefton M, Oliva-Moreno J (2017) Social/economic costs and health-related quality of life in patients with spinal muscular atrophy (SMA) in Spain. Orphanet J Rare Dis. https://doi.org/10.1186/ s13023-017-0695-0

39. Dunaway Young S, Montes J, Kramer SS, Marra J, Salazar R, Cruz R, Chiriboga CA, Garber CE, De Vivo DC (2016) Sixminute walk test is reliable and valid in spinal muscular atrophy. Muscle Nerve 54:836-842. https://doi.org/10.1002/mus.25120

40. Main M, Kairon H, Mercuri E, Muntoni F (2003) The Hammersmith functional motor scale for children with spinal muscular atrophy: a scale to test ability and monitor progress in children with limited ambulation. Eur J Paediatr Neurol 7:155-159. https ://doi.org/10.1016/s1090-3798(03)00060-6

41. O'Hagen JM, Glanzman AM, McDermott MP, Ryan PA, Flickinger J, Quigley J, Riley S, Sanborn E, Irvine C, Martens WB, Annis C, Tawil R, Oskoui M, Darras BT, Finkel RS, De Vivo DC (2007) An expanded version of the Hammersmith Functional Motor Scale for SMA II and III patients. Neuromuscul Disord 17:693-697. https://doi.org/10.1177/0883073811420294

42. Mazzone ES, Mayhew A, Montes J, Ramsey D, Fanelli L, Young SD, Salazar R, De Sanctis R, Pasternak A, Glanzman A, Coratti G, Civitello M, Forcina N, Gee R, Duong T, Pane M, Scoto M, Pera MC, Messina S, Tennekoon G, Day JW, Darras BT, De Vivo DC, Finkel R, Muntoni F, Mercuri E (2017) Revised upper limb module for spinal muscular atrophy: development of a new module. Muscle Nerve 55:869-874. https://doi.org/10.1002/ mus. 25430

43. von Elm E, Altman DG, Egger M, Pocock SJ, Gotzsche PC, Vandenbroucke JP, Initiative STROBE (2014) The Strengthening the Reporting of Observational Studies in Epidemiology (STROBE) Statement: guidelines for reporting observational studies. Int J Surg 12:1495-1499. https://doi.org/10.1016/j.ijsu.2014.07.013

44. Montes J, McDermott MP, Mirek E, Mazzone ES, Main M, Glanzman AM, Duong T, Young SD, Salazar R, Pasternak A, Gee R, De Sanctis R, Coratti G, Forcina N, Fanelli L, Ramsey D, Milev E, Civitello M, Pane M, Pera MC, Scoto M, Day JW, Tennekoon G, Finkel RS, Darras BT, Muntoni F, De Vivo DC, Mercuri E (2018) Ambulatory function in spinal muscular atrophy: agerelated patterns of progression. PLoS ONE 13:e0199657. https:// doi.org/10.1371/journal.pone.0199657

45. Kizina K, Stolte B, Totzeck A, Bolz S, Schlag M, Ose C, von Velsen O, Kleinschnitz C, Hagenacker T (2020) Fatigue in adults with spinal muscular atrophy under treatment with nusinersen. Sci Rep. https://doi.org/10.1038/s41598-020-68051-w

46. Schwarz R, Krauss O, Hinz A (2003) Fatigue in the general population. Onkologie 26:140-144. https://doi.org/10.1159/000069834

47. Lerdal A, Wahl A, Rustoen T, Hanestad BR, Moum T (2005) Fatigue in the general population: a translation and test of the psychometric properties of the Norwegian version of the fatigue severity scale. Scand J Public Health 33:123-130. https://doi. org/10.1080/14034940410028406

48. Gibbons CJ, Mills RJ, Thornton EW, Ealing J, Mitchell JD, Shaw PJ, Talbot K, Tennant A, Young CA (2011) Development of a patient reported outcome measure for fatigue in motor neurone disease: the Neurological Fatigue Index (NFI-MND). Health Qual Life Outcomes. https://doi.org/10.1186/1477-7525-9-101

49. Lou JS, Reeves A, Benice T, Sexton G (2003) Fatigue and depression are associated with poor quality of life in ALS. Neurology 60:122-123. https://doi.org/10.1212/01.wnl.0000042781.22278 $.0 \mathrm{a}$

50. Ghajarzadeh M, Jalilian R, Eskandari G, Sahraian MA, Azimi A, Mohammadifar M (2013) Fatigue in multiple sclerosis: relationship with disease duration, physical disability, disease pattern, age and sex. Acta Neurol Belg 113:411-414. https://doi.org/10.1007/ s13760-013-0198-2

51. Zuo LJ, Yu SY, Hu Y, Wang F, Piao YS, Lian TH, Yu QJ, Wang RD, Li LX, Guo P, Du Y, Zhu RY, Jin Z, Wang YJ, Wang XM, Chan P, Chen SD, Wang YJ, Zhang W (2016) Serotonergic dysfunctions and abnormal iron metabolism: Relevant to mental fatigue of Parkinson disease. Sci Rep. https://doi.org/10.1038/ s41598-016-0018-Z

52. Van Der Slot WM, Nieuwenhuijsen C, Van Den Berg-Emons RJ, Bergen MP, Hilberink SR, Stam HJ, Roebroeck ME (2012) Chronic pain, fatigue, and depressive symptoms in adults with spastic bilateral cerebral palsy. Dev Med Child Neurol 54:836842. https://doi.org/10.1111/j.1469-8749.2012.04371.x

53. Kratz AL, Murphy SL, Braley TJ (2017) Pain, fatigue, and cognitive symptoms are temporally associated within but not across days in multiple sclerosis. Arch Phys Med Rehabil 98:2151-2159. https://doi.org/10.1016/j.apmr.2017.07.003

54. Hagelin CL, Wengstrom Y, Runesdotter S, Furst CJ (2007) The psychometric properties of the Swedish Multidimensional Fatigue Inventory MFI-20 in four different populations. Acta Oncol 46:97-104. https://doi.org/10.1080/02841860601009430

55. Montes J, Dunaway Young S, Mazzone ES, Pasternak A, Glanzman AM, Finkel RS, Darras BT, Muntoni F, Mercuri E, De Vivo DC, Bishop KM, Schneider E, Bennett CF, Foster R, Farwell W, CS2, and CS12 Study Groups (2019) Nusinersen improves walking distance and reduces fatigue in later-onset spinal muscular 
atrophy. Muscle Nerve 60:409-414. https://doi.org/10.1002/ mus. 26633

56. Holzner B, Kemmler G, Greil R, Kopp M, Zeimet A, Raderer M, Hejna M, Zochbauer S, Krajnik G, Huber H, Fleischhacker WW, Sperner-Unterweger B (2002) The impact of hemoglobin levels on fatigue and quality of life in cancer patients. Ann Oncol 13:965-973. https://doi.org/10.1093/annonc/mdf122

57. Mongiovi P, Dilek N, Garland C, Hunter M, Kissel JT, Luebbe E, McDermott MP, Johnson N, Heatwole C (2018) Patient reported impact of symptoms in spinal muscular atrophy (PRISM-SMA). Neurology 91:e1206-e1214. https://doi.org/10.1212/WNL.00000 00000006241

\section{Affiliations}

\section{Camilla Binz ${ }^{1}$. Olivia Schreiber-Katz ${ }^{1} \cdot$ Mareike Kumpe $^{1} \cdot$ Gresa Ranxha $^{1} \cdot$ Hannah Siegler ${ }^{1} \cdot$ Gary Wieselmann $^{1}$. Susanne Petri ${ }^{1} \cdot$ Alma Osmanovic $^{1}$}

Camilla Binz

binz.camilla@mh-hannover.de

Mareike Kumpe

kumpe.mareike@mh-hannover.de

Gresa Ranxha

ranxha.gresa@mh-hannover.de

Hannah Siegler

siegler.hannah@mh-hannover.de
Gary Wieselmann

gary.wieselmann@stud.mh-hannover.de

Susanne Petri

petri.susanne@mh-hannover.de

Alma Osmanovic

osmanovic.alma@mh-hannover.de

1 Department of Neurology, Hannover Medical School, Carl-Neuberg-Strasse 1, 30625 Hannover, Germany 